\title{
El Problema del Voceador con Incertidumbre Paramétrica
}

\author{
David F. Muñoz ${ }^{(1)}$, David G. Muñoz ${ }^{(2)}$ y Adán Ramírez-López ${ }^{(1)}$ \\ (1) Dpto. de Ingeniería Industrial y de Operaciones, Instituto Tecnológico Autónomo de México, \\ Río Hondo 1, 01080 México Distrito Federal, México \\ (2) Research and Development, AOL Advertising, 395 Page Mill Road, Palo Alto CA 94404, USA \\ (e-mail: davidm@itam.mx; davidmm82@gmail.com; adan.ramirez@itam.mx)
}

Recibido Nov. 18, 2015; Aceptado Ene. 20, 2016; Versión final Feb. 27, 2016, Publicado Ago. 2016

\begin{abstract}
Resumen
En este artículo se presenta la formulación y solución del problema del voceador bajo un enfoque bayesiano. Este enfoque permite incorporar la incertidumbre en los parámetros de la distribución de la demanda (que es inducida por el proceso de estimación de dichos parámetros). Se presenta un ejemplo que tiene solución analítica y se conducen experimentos con dicho modelo para comparar los resultados del método bayesiano propuesto con los que se obtienen al aplicar el método clásico. Además, se calcula el tamaño óptimo de pedido utilizando simulación estocástica, método que se aconseja usar cuando la complejidad del modelo no permite la obtención de una solución analítica.
\end{abstract}

Palabras clave: problema del voceador; administración de inventarios; incertidumbre paramétrica; pronóstico bayesiano; simulación estocástica.

\section{The Newsvendor Problem under Parametric Uncertainty}

\begin{abstract}
The formulation and solution to the newsvendor problem using a Bayesian approach is presented. This approach allows us to incorporate the uncertainty in the parameters of demand distribution (introduced in the process of parameter estimation). An example that allows obtaining an analytical solution is presented and experiments are performed with the model in order to compare the results using the proposed Bayesian approach versus the classical method. In addition, the optimal order size is determined using stochastic simulation, methodology that is suggested when model complexity does not allow obtaining an analytical solution.
\end{abstract}

Keywords: newsvendor problem; inventory management; parameter uncertainty; bayesian forecasting; stochastic simulation. 


\section{INTRODUCCIÓN}

Sea $D$ la demanda (durante el periodo de venta) de un artículo de temporada. Si $w \geq 0$ denota la pérdida por unidad no vendida al final del periodo, y $u \geq 0$ denota el beneficio por cada unidad vendida en el periodo, el beneficio que se obtiene para un tamaño de pedido de $Q$ unidades es:

$$
b(Q)=\left\{\begin{array}{cc}
u D-w(Q-D), & D<Q, \\
u Q, & D \geq Q
\end{array}\right.
$$

El procedimiento más difundido (ver, por ejemplo, Muñoz, 2009) para encontrar el tamaño óptimo de pedido $Q_{C}$ consiste en considerar una función de densidad $f(y \mid \theta)$ (el análisis es similar para el caso discreto) para la demanda $D$, donde $\theta$ es un vector de parámetros y, asumiendo que $\theta$ es conocido, se considera el beneficio esperado

$$
B_{C}(Q \mid \theta) \stackrel{\text { def }}{=} E[b(Q) \Theta=\theta]=u \int_{0}^{Q} y f(y \mid \theta) d y-w \int_{0}^{Q}(Q-y) f(y \mid \theta) d y+u Q \int_{Q}^{\infty} f(y \mid \theta) d y .
$$

Esta función tiene derivada para $Q>0$ y se puede encontrar el óptimo igualando la primera derivada a cero, en cuyo caso se encontrará que el tamaño óptimo de pedido $Q_{C}$ que maximiza $B_{C}(Q \mid \theta)$ satisface

$$
F_{C}\left(Q_{C} \mid \theta\right)=\int_{0}^{Q_{C}^{*}} f(y \mid \theta) d y=\frac{u}{u+w}
$$

Notar que $F_{C}(y \mid \theta)$ es la función de distribución acumulativa (fda) de la demanda dado $[\Theta=\theta]$. Además, si $f(x \mid \theta)>0$ es continua en una vecindad de $Q_{C}$, la condición (3) es suficiente para encontrar el valor óptimo $Q_{C}$. Nótese también que hemos utilizamos el subíndice $C$ para hacer referencia a la solución del problema del voceador bajo el método clásico descrito en los libros de texto (e.g., Muñoz, 2009).

En la práctica, el valor de $\theta$ se estima a partir de un conjunto de datos $\mathrm{x}=\left(\mathrm{x}_{1}, \ldots, \mathrm{x}_{\mathrm{n}}\right)$, por ejemplo, tomando el estimador (máximo verosímil) que maximiza una función de verosimilitud $L(\theta \mid x)$. Actualmente, el procedimiento más difundido para determinar el tamaño óptimo de pedido consiste en imponer (3) para $\theta=\hat{\theta}$, donde $\hat{\theta}=\hat{\theta}(x)$ es un estimador puntual. Aunque este procedimiento se encuentra ampliamente difundido en los textos de Administración de Operaciones, tiene el inconveniente de asumir que el estimador puntual es igual al parámetro, por lo que en este artículo presentamos un enfoque Bayesiano del problema del voceador (i.e., encontrar el tamaño óptimo de pedido) incorporando la incertidumbre (que introduce el proceso de estimación) en el valor del vector de parámetros.

La Estadística Bayesiana ha mostrado su utilidad en diversas áreas, incluyendo el análisis de procesos industriales (ver, por ejemplo, Limón et al., 2012), ya que posibilita el análisis de problemas que no se pueden atacar con la Estadística "frecuentista". En el caso de la administración de inventarios, los métodos Bayesianos para introducir la incertidumbre paramétrica han sido propuestos desde el trabajo pionero de Scarf (1959), donde el autor discute la optimalidad de una regla de actualización Bayesiana para administrar inventarios. Silver (1965) propone la incorporación de la incertidumbre paramétrica usando métodos Bayesianos y muestra cómo calcular el punto de reorden modelando la demanda como una distribución multinomial. Muchas reglas Bayesianas para actualizar las políticas para la administración de inventarios (con base en nueva información sobre la demanda) han sido propuestos en la literatura, véase por ejemplo, Azouri (1985), Eppen e lyer (1997), Lariviere y Porteus (1999), Chen y Plambeck (2008), Chen (2010), Jain et al. (2015), y las referencias de estos artículos.

También se han propuesto métodos para incorporar la incertidumbre paramétrica usando un enfoque "frecuentista" (i.e., reemplazando el valor del parámetro desconocido por su estimador); por ejemplo, Cheng y Holland (2004) proponen el re-muestreo (usando simulación) de observaciones de una distribución conjunta donde el parámetro ha tomado el valor del estimador. Bajo este mismo enfoque, también se ha propuesto un método no paramétrico bajo el cual, si se dispone de observaciones que pueden asumirse como una muestra aleatoria de la demanda $D$, la solución (3) del problema se aproxima por el correspondiente cuantil de la muestra aleatoria. La calidad de esta aproximación depende, como es de esperar, del tamaño de la muestra disponible y se discute en Levi et al. (2007) y Levi et al. (2015). Como hacemos explícito en la siguiente sección, nuestro enfoque requiere de la existencia de datos relacionados con la demanda, pero no tiene la limitación de requerir, necesariamente, de una muestra aleatoria de la 
demanda futura, por lo que se puede aplicar en situaciones más generales (e.g., para administrar inventarios de productos nuevos o de temporada). La metodología utilizada en este artículo es similar a la de Jain et al. (2015), donde los autores muestran que, en particular, se puede aplicar con datos sólo de ventas (desconociendo el faltante) o de tiempos entre ocurrencias de la demanda (como en el ejemplo mostrado más adelante en este artículo). Por otro lado, también se han propuesto extensiones del problema del voceador; por ejemplo, Agrawal y Seshadri (2000) estudian la incertidumbre en el precio y el tamaño del pedido, mientras que Keren y Pliskin (2006) estudian el efecto de la aversión al riesgo. En nuestro conocimiento, en ninguno de estos trabajos, ni en la bibliografía relacionada, se discute el uso de la simulación para resolver el problema del voceador con incertidumbre en los parámetros de la distribución de la demanda.

Luego de esta Introducción, en la siguiente sección describimos los fundamentos de este enfoque Bayesiano, que permite incorporar la incertidumbre paramétrica en el problema del voceador. A continuación, en la siguiente sección, presentamos un ejemplo sencillo, que además nos permite diseñar un procedimiento computacional para encontrar el tamaño óptimo de pedido sin recurrir a la simulación. Posteriormente aprovechamos este ejemplo sencillo para ilustrar cómo se puede estimar el tamaño óptimo de pedido (en problemas más complicados) usando simulación, así como presentamos una comparación empírica de los resultados que se obtienen bajo el método clásico, en contraste con los resultados que se obtienen utilizando la metodología Bayesiana propuesta. Finalmente, en la última sección presentamos nuestras conclusiones y recomendaciones.

\section{METODOLOGÍA}

Bajo un enfoque Bayesiano, el vector de parámetros es una variable aleatoria $\Theta$ que tiene una función de densidad a priori $p(\theta)$, por lo que la densidad posterior (dado el conjunto de datos $x$ ) resulta

$p(\theta \mid x)=\frac{p(\theta) L(x \mid \theta)}{\int_{S_{0}} p(\theta) L(x \mid \theta) d \theta}$,

donde $x \in \mathfrak{R}^{d}, \theta \in S_{0}$ y $L(x \mid \theta)$ es la función de verosimilitud. A partir de (4), bajo la misma notación utilizada en (3), la fda de la demanda (dado $[X=x]$ ) resulta

$$
F_{B}(y \mid x)=E\left[E\left[F_{C}(y \mid \Theta)\right] X=x\right]=\int_{S_{0}} F_{C}(y \mid \theta) p(\theta \mid x) d \theta
$$

para $y \geq 0$, donde $F_{C}(y \mid \theta)$ y $p(\theta \mid x)$ están definidas en (3) y (4), respectivamente. Notar que, bajo una notación más difundida (ver, por ejemplo, el teorema 9.1.2 de Chung, 2001) se tiene que $E\left[F_{C}(y \mid \Theta)\right]=E[[D \leq y] \Theta]$, donde $[[D \leq y]$ es uno cuando ocurre el evento $[D \leq y]$ y cero de otra forma.

Similarmente, a partir de (1) se obtiene el beneficio esperado (dado $[X=x]$ )

$$
B_{B}(Q \mid x)=u \int_{0}^{Q} y d F_{B}(y \mid x)-w \int_{0}^{Q}(Q-y) d F_{B}(y \mid x)+u Q \int_{Q}^{\infty} d F_{B}(y \mid x),
$$

donde $F_{B}(y \mid x)$ es la fda definida en (5), lo que muestra que $B_{B}(Q \mid x)$ tiene una forma similar a $B_{C}(Q \mid x)$ definida en (2) y, en consecuencia, el tamaño óptimo de pedido $Q_{B}$ considerando la incertidumbre paramétrica satisface

$$
F_{B}\left(Q_{B} \mid x\right)=\frac{u}{u+w}
$$

donde $F_{B}(y \mid x)$ está definida en (5). Notar que el subíndice $B$ en las ecuaciones (5), (6) y (7) hace referencia a la solución del problema del voceador bajo un enfoque Bayesiano.

Es conveniente mencionar que nuestra formulación es diferente de la que se presenta en Jain et al. (2015), donde los autores proponen un modelo de programación dinámica para resolver el problema del voceador bajo un enfoque multi-periodo, con la finalidad de mostrar las ventajas de utilizar una regla de actualización Bayesiana más eficiente. La diferencia fundamental es que en este artículo consideramos un beneficio 
esperado (de un solo periodo) que depende explícitamente del conjunto de datos disponibles $x$. Esta formulación permite obtener una solución simple en la forma de (7).

En el caso en que la demanda sea discreta, tomando valores $d_{1}<d_{2}<\ldots$, la función $F_{B}(y \mid x)$ no es continua, y la ecuación (7) podría no tener solución, en cuyo caso debe buscarse el valor $d_{k}$ que satisface:

$$
P\left[D \leq d_{k} \mid X=x\right] \leq \frac{u}{u+w} \leq P\left[D \leq d_{k+1} \mid X=x\right]
$$

para evaluar $B_{B}\left(d_{k} \mid x\right)$ y $B_{B}\left(d_{k+1} \mid x\right)$, donde:

$$
B_{B}(Q \mid x)=u \sum_{j \leq Q} j P[D=j \mid X=x]-w \sum_{j \leq Q}(Q-j) P[D=j \mid X=x]+u Q P[D>Q \mid X=x]
$$

si $B_{B}\left(d_{k} \mid x\right) \geq B_{B}\left(d_{k+1} \mid x\right)$, el óptimo será $Q_{B}=d_{k}$, de otra forma $Q_{B}=d_{k+1}$. Nótese que (8) es el equivalente de (7) para el caso discreto, y que en ambas ecuaciones no se está considerando ningún costo fijo por ordenar. Si existe un inventario inicial $Q_{0}$, no se debe ordenar cuando $Q_{0} \geq Q_{B}$, y de otra forma se deben ordenar $Q_{B}-Q_{0}$ unidades sólo si $B_{B}\left(Q_{B} \mid x\right)-B_{B}\left(Q_{0} \mid x\right)>C_{0}$, donde $C_{0}$ es el costo fijo por ordenar.

\section{EJEMPLO ILUSTRATIVO}

A continuación ilustramos la aplicación de la metodología propuesta a través de una variante del modelo presentado en Muñoz y Muñoz (2009) para el pronóstico de demanda intermitente. Se sabe que la demanda de clientes para una tienda de repuestos ocurre según un proceso de Poisson, aunque existe incertidumbre en la tasa de llegadas $\Theta_{0}$, de manera que, dado $\left[\Theta_{0}=\theta_{0}\right]$, los tiempos entre las llegadas sucesivas de clientes son independientes e idénticamente distribuidos (i.i.d.) con función de densidad exponencial:

$$
f\left(y \mid \theta_{0}\right)=\left\{\begin{array}{cc}
\theta_{0} e^{-\theta_{0} y}, & y>0 \\
0, & \text { de otra forma }
\end{array}\right.
$$

donde $\theta_{0} \in(0, \infty)$. Cada cliente puede ordenar $j$ unidades de cierto repuesto con probabilidad $\Theta_{1 j}, j=1, \ldots, q$, $q \geq 2$, siendo que, bajo un enfoque Bayesiano, existe incertidumbre en el valor de estas probabilidades. Denotando $\Theta_{1}=\left(\Theta_{11}, \ldots, \Theta_{1(q-1)}\right)$ y $\Theta_{1 q}=1-\sum_{j=1}^{q-1} \Theta_{1 j}$, entonces $\Theta=\left(\Theta_{0}, \Theta_{1}\right)$ es el vector de parámetros, y el espacio de parámetros resulta $S_{0}=(0, \infty) \otimes S_{01}$, donde

$$
S_{01}=\left\{\left(\theta_{11}, \ldots, \theta_{1(q-1)}\right): \sum_{j=1}^{q-1} \theta_{1 j} \leq 1 ; \theta_{1 j} \geq 0, j=1, \ldots, q-1\right\} \text {. }
$$

La demanda total durante un periodo de longitud $T$ está dada por

$$
D=\left\{\begin{array}{cc}
N(T) & \\
\sum_{i=1} U_{i}, & N(T)>0 \\
0, & \text { de otra forma }
\end{array}\right.
$$

donde $N(s)$ es el número de clientes que llegaron en el intervalo $[0, s], s \geq 0$, y $U_{1}, U_{2} \ldots$ son las demandas individuales (asumidas condicionalmente independientes en relación a $\Theta$ ). La información sobre $\Theta$ consiste de observaciones (i.i.d.) $v=\left(v_{1}, \ldots, v_{n}\right), v=\left(u_{1}, \ldots, u_{n}\right)$ de clientes pasados, donde $v_{i}$ es el tiempo entre la llegada del cliente $i$ y el anterior $(i-1)$, y $u_{i}$ es el número de unidades ordenadas por el cliente $i$. Las funciones de verosimilitud para $v$ y $v$ tienen la forma

$$
L\left(v \mid \theta_{0}\right)=\theta_{0}^{n} e^{-\theta_{0} \sum_{i=1}^{n} v_{i}}, \text { y } L\left(v \mid \theta_{1}\right)=\left(1-\sum_{j=1}^{q-1} \theta_{1 j}\right)^{c_{q}} \prod_{j=1}^{q-1} \theta_{1 j}^{c_{j}},
$$

respectivamente, donde $\theta_{1}=\left(\theta_{11}, \ldots, \theta_{1(q-1)}\right)$, y $c_{j}=\sum_{i=1}^{n} l\left[u_{i}=j\right]$ es el número de clientes que ordenaron $j$ unidades. 
Bajo un punto de vista objetivo, podemos considerar una densidad a priori no informativa para $\Theta$, utilizando la densidad de Jeffrey. Como es sabido (ver, e.g., Bernardo, 2009), la densidad de Jeffrey para el modelo exponencial es $p\left(\theta_{0}\right)=\theta_{0}^{-1}, \theta_{0} \in(0, \infty)$, por lo que sigue de (4) y (12) que

$p\left(\theta_{0} \mid v\right)=\frac{\theta_{0}^{n-1}\left(\sum_{i=1}^{n} v_{i}\right)^{n} e^{-\theta_{0} \sum_{i=1}^{n} v_{i}}}{(n-1) !}$,

que corresponde a la distribución Gamma $\left(n, \sum_{i=1}^{n} v_{i}\right)$, donde $\operatorname{Gamma}\left(\beta_{1}, \beta_{2}\right)$ denota a una distribución gamma con esperanza $\beta_{1} \beta_{2}^{-1}$. Similarmente, la densidad de Jeffrey para el modelo multinomial (ver, e.g., Berger y Bernardo, 1992) corresponde a la distribución de Dirichlet:

$p\left(\theta_{1}\right)=\frac{\left(1-\sum_{j=1}^{q-1} \theta_{1 j}\right)^{-1 / 2} \prod_{j=1}^{q-1} \theta_{1 j}^{-1 / 2}}{B(1 / 2, \ldots, 1 / 2)}$,

donde $B\left(a_{1}, \ldots, a_{q}\right)=\prod_{j=1}^{q} \Gamma\left(a_{j}\right) / \Gamma\left(\sum_{j=1}^{q} a_{j}\right)$, para $a_{1}, \ldots, a_{q}>0$, por lo que sigue de (4) y (12) que

$p\left(\theta_{1} \mid v\right)=\frac{\left(1-\sum_{j=1}^{q-1} \theta_{1 j}\right)^{c_{q}-1 / 2} \prod_{j=1}^{q-1} \theta_{1 j}^{c_{j}-1 / 2}}{B\left(c_{1}+1 / 2, \ldots, c_{q}+1 / 2\right)}$,

es decir, corresponde a una distribución de Dirichle $\left(c_{1}+1 / 2, \ldots, c_{q}+1 / 2\right)$. Denotando $x_{i}=\left(v_{i}, u_{i}\right)$, $i=1, \ldots, n, \quad x=\left(x_{1}, \ldots, x_{n}\right), \quad \theta=\left(\theta_{0}, \theta_{1}\right), \quad y$ asumiendo independencia, la densidad posterior resulta $p(\theta \mid x)=p\left(\theta_{0} \mid v\right) p\left(\theta_{1} \mid v\right)$, donde $p\left(\theta_{0} \mid v\right)$ y $p\left(\theta_{1} \mid v\right)$ están definidas en (13) y (15), respectivamente.

Notar que, en este ejemplo, se puede obtener una expresión analítica para el estimador puntual de la demanda $\mu=E[D \mid X=x]$, ya que de (13) y (15) se obtienen $E\left[\Theta_{0} \mid V=v\right]=n\left(\sum_{i=1}^{n} v_{i}\right)^{-1}$ y $E\left[\Theta_{1 j} \mid U=v\right]=c^{-1}\left(c_{j}+1 / 2\right)$ (donde $\left.c=\sum_{j=1}^{n}\left(c_{j}+1 / 2\right)=n+q / 2\right)$, por lo que sigue de (11) que

$$
\begin{aligned}
\mu & =E[E[D \mid \Theta, X=x] X=x]=E\left[E[N(T) \mid \Theta] E\left[U_{1} \mid \Theta\right] X=x\right] \\
& =T E\left[\Theta_{0} \sum_{j=1}^{q} j \Theta_{1 j} \mid X=x\right]=T E\left[\Theta_{0} \mid V=v\right] \sum_{j=1}^{q} j E\left[\Theta_{1 j} \mid U=v\right]=T n\left(\sum_{j=1}^{n} v_{i}\right)^{-1}(n+q / 2)^{-1} \sum_{j=1}^{q} j\left(c_{j}+1 / 2\right),
\end{aligned}
$$

expresión que nos permite calcular el pronóstico puntual de la demanda $\mu$ a partir del conjunto de datos $x$. En este caso, sin embargo, no es sencillo obtener una expresión analítica para la fda y el tamaño óptimo de pedido, por lo que se puede aplicar el algoritmo de muestreo posterior (MP) descrito en la sección 3.1 de Muñoz et al. (2013) para calcular, por medio de la simulación, el correspondiente tamaño óptimo de pedido, dado un nivel de servicio $\alpha=u /(u+w)$. Como ilustraremos en la siguiente sección, bajo el algoritmo MP se propone utilizar la distribución posterior definida en (4) para generar (por simulación) una muestra aleatoria de la demanda, estimando el correspondiente cuantil de la demanda a partir de esta muestra. Nótese que, tanto en Muñoz et al. (2013) como en Levi et al. (2015) se propone estimar el cuantil de la distribución de la demanda por medio del cuantil de una muestra aleatoria; sin embargo, dichos artículos abordan problemas diferentes.

Para el caso en que $q=1$ (i.e., cada cliente demanda siempre una unidad del repuesto), el modelo se simplifica y no es necesario recurrir a la simulación para encontrar el tamaño óptimo de pedido. En este caso, podemos ignorar a $\theta_{1}$ y los valores $u_{i}$ (que son siempre iguales a 1). Denotando $x=v, \theta=\theta_{0}$, tenemos que $P[D=j \mid \Theta=\theta]=P[N(T)=j \mid \Theta=\theta]=e^{-\theta T}(\theta T)^{j} / j !$, y tomando en cuenta (13) se obtiene 


$$
P[D=j \mid X=x]=(n+j-1)\left(\frac{\sum_{i=1}^{n} x_{i}}{T+\sum_{i=1}^{n} x_{i}}\right)^{n}\left(\frac{T}{T+\sum_{i=1}^{n} x_{i}}\right)^{j},
$$

Para $j=0$. 1. ..., expresión que corresponde a una distribución binomial negativa. Utilizando las ecuaciones (8), (9) y (17) se puede determinar el tamaño óptimo de pedido $Q_{B}$ para este caso particular, sin necesidad de recurrir al algoritmo MP ni a la simulación.

\section{RESULTADOS EXPERIMENTALES}

Con la finalidad de ilustrar la validez del algoritmo MP y, en particular, cómo se puede aplicar para determinar el tamaño óptimo de pedido, utilizaremos el ejemplo de la sección anterior que tiene la solución analítica (17) para la distribución posterior de la demanda. En primer lugar, hemos considerado los datos $T=15, n=20, \sum_{i=1}^{n} x_{i}=10, u=9, w=1$. Con estos datos, el nivel de servicio óptimo es $\alpha=u /(u+w)=0.9$, y luego de aplicar el método Bayesiano descrito por la ecuaciones (8) y (9), y la distribución posterior definida en (17), obtuvimos un tamaño óptimo de pedido $Q_{B}=41$, con un beneficio esperado, de acuerdo con (6), de $B_{B}\left(Q_{B} \mid x\right)=253.38$, y un nivel de servicio de $F_{B}\left(Q_{B} \mid x\right)=0.901$ (ligeramente superior a 0.9 ).

Con el objetivo de comparar los resultados obtenidos con el método clásico, nótese que, a partir de (11) se puede deducir que la fda $F_{C}(y \mid \theta)$ definida en (3) corresponde a la distribución de Poisson con esperanza $\theta T$ y, por otro lado, el estimador máximo verosímil de $\theta$ es $\hat{\theta}=2$, por lo que, al aplicar el método clásico, imponiendo condiciones similares a (8) y (9), se obtuvo $Q_{C}=37$, reportando un beneficio esperado, de acuerdo con (2), de $B_{C}\left(Q_{C} \mid x\right)=260.05>B_{B}\left(Q_{B} \mid x\right)$, y un nivel de servicio, de acuerdo con la distribución

posterior (17), de $F_{B}\left(Q_{C} \mid x\right)=0.803<F_{B}\left(Q_{B} \mid x\right)$. Estos resultados sugieren que el método clásico ha exagerado el beneficio esperado, a la vez que proporciona un nivel de servicio más conservador que el método Bayesiano, confirmando la intuición de que la incertidumbre paramétrica introduce una distribución posterior $F_{B}(y \mid x)$ de la demanda que tiene mayor dispersión que la del método clásico $F_{C}(y \mid x)$. A continuación presentamos resultados empíricos que confirman estas observaciones. Más adelante ilustraremos también cómo se puede estimar el tamaño óptimo de pedido cuando no se puede (o es muy complicado) encontrar una solución analítica.

\section{Comparación Empírica del Método Clásico con el Método Bayesiano}

En un primer experimento, asumimos que el valor de la tasa de llegadas de los clientes es de $\theta=2$ y generamos $m=1000$ muestras de tiempos de llegadas de tamaño $n=20$ cada una. Para cada una de estas muestras calculamos $\sum_{i=1}^{n} x_{i}$ y los tamaños óptimos de pedido (clásico y Bayesiano) con los datos anteriores $(T=15, n=20, u=9, w=1)$. Para cada una de las 1000 repeticiones calculamos el exceso del beneficio esperado $B_{C}\left(Q_{C} \mid x\right)-B_{B}\left(Q_{B} \mid x\right)$, y el nivel de servicio del tamaño óptimo de pedido clásico $F_{B}\left(Q_{C} \mid x\right)$.

Como puede observarse de la Fig. 1, el método clásico ha exagerado el beneficio esperado en todas las repeticiones del experimento, y similarmente, de la Fig. 2 se puede también apreciar que el método clásico ha proporcionado un nivel de servicio más conservador en todas las repeticiones del experimento. Este último resultado, sin embargo, parece ser un error de muestreo, ya que debe existir una probabilidad positiva (en este caso, muy pequeña) de que el tamaño óptimo de pedido clásico $Q_{C}$ sea lo suficientemente grande para proporcionar un nivel de servicio $F_{B}\left(Q_{C} \mid x\right)$ mayor que el deseado $(\alpha)$; en la Fig. 3 ilustramos este caso, donde se muestran los resultados del mismo experimento de la Fig. 2, cambiando únicamente el tamaño de muestra a $n=300$.

Con el objetivo de remarcar que los resultados con el método Bayesiano propuesto son consistentes con los resultados del método clásico, replicamos los experimentos anteriores considerando diferentes tamaños de muestra para los tiempos entre llegadas de los clientes y se han reportado los resultados en la Tabla 1. Como puede apreciarse de la tabla, a medida que el tamaño de muestra crece, el exceso de beneficio tiende a cero y el nivel de servicio tiende al óptimo de 0.9 , mostrando que ambos métodos coinciden a medida que el tamaño de muestra crece (y la incertidumbre paramétrica es despreciable). 


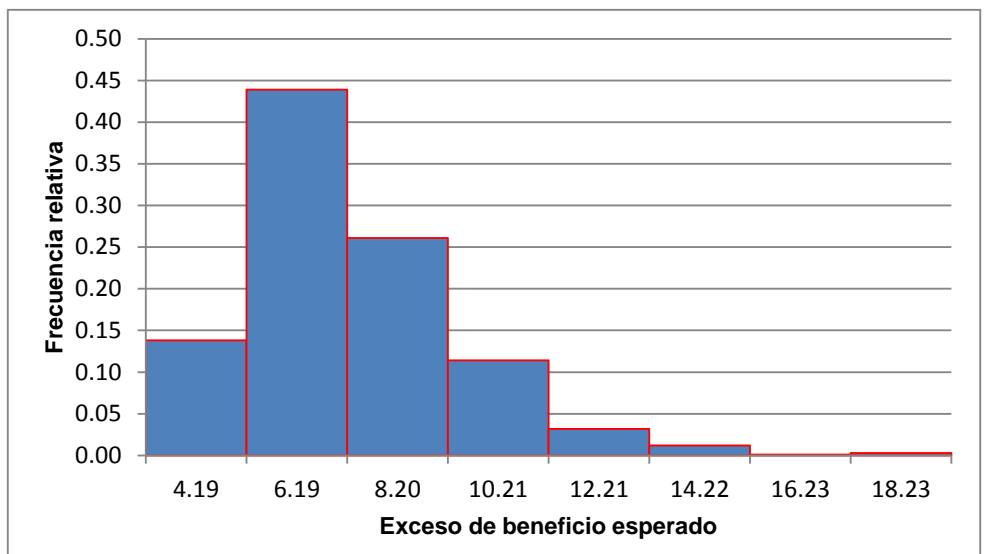

Fig. 1. Histograma de las diferencias $B_{C}\left(Q_{C} \mid X\right)-B_{B}\left(Q_{B} \mid X\right)$ con base en 1000 repeticiones de los experimentos de estimación clásica y Bayesiana.

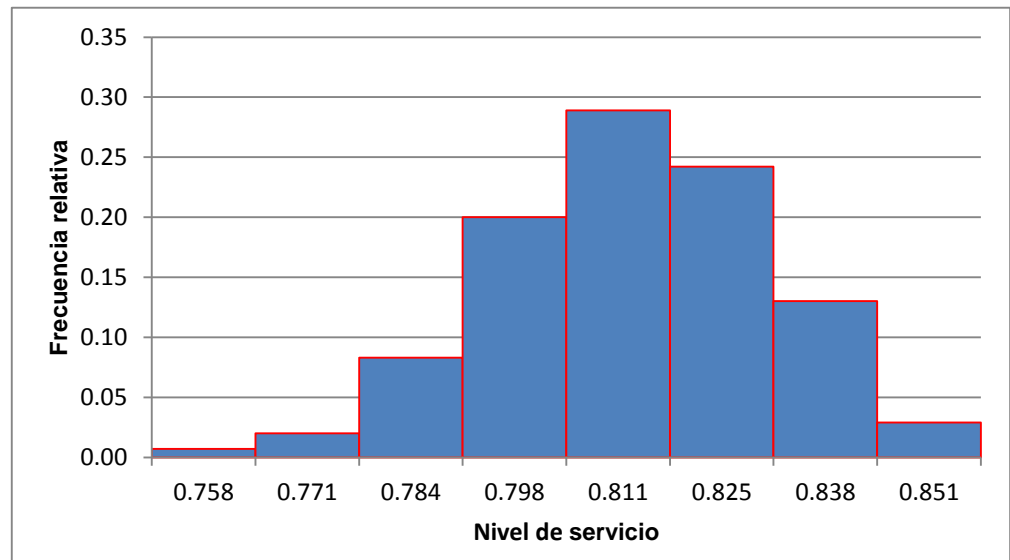

Fig. 2. Histograma del verdadero nivel de servicio $F_{B}\left(Q_{C} / X\right)$ del tamaño óptimo de pedido clásico con base en 1000 repeticiones de los experimentos de estimación clásica y Bayesiana con $n=50$.

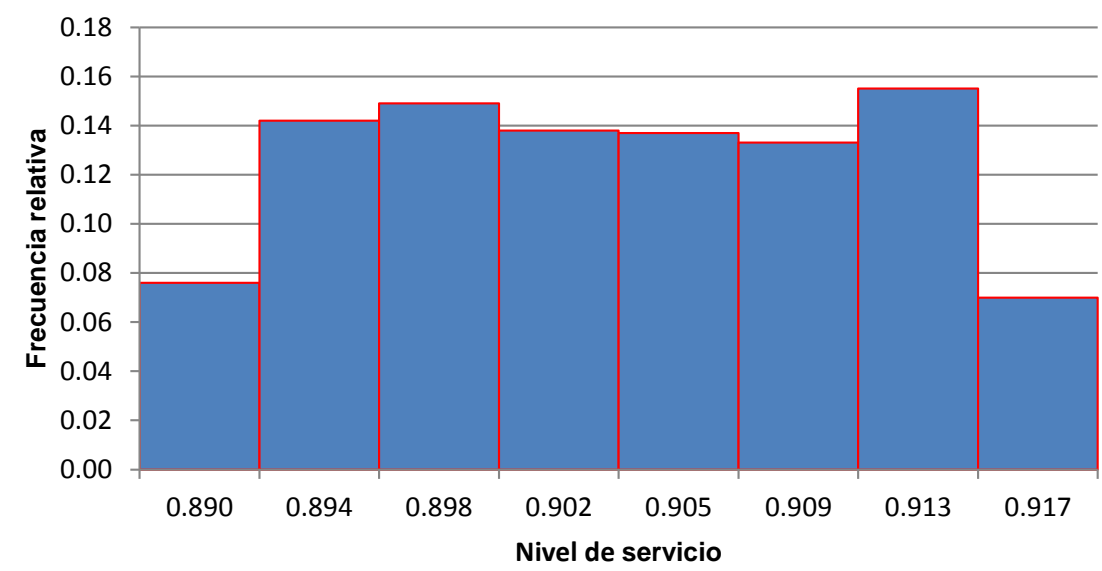

Fig. 3. Histograma del verdadero nivel de servicio $F_{B}\left(Q_{C} / X\right)$ del tamaño óptimo de pedido clásico con base en 1000 repeticiones de los experimentos de estimación clásica y Bayesiana con $n=$ 300 .

\section{Análisis de Algunos Resultados Experimentales}

A continuación analizamos la posibilidad de que los resultados reportados en la Fig. 1 puedan generalizarse para otros valores de los parámetros que hemos considerado en los experimentos correspondientes. Con este objetivo, consideremos dado el valor de $\bar{x}$ (luego de haber simulado una muestra de tamaño $n$ de la distribución exponencial con esperanza $1 / \theta)$ y sean $Y, Z$ variables aleatorias distribuidas como Poisson con esperanza $\lambda=T / \bar{x}$ y como binomial negativa con la función de probabilidades definida en (17), es decir, con parámetros $n$ y $p=\bar{x} /(\bar{T}+\bar{x})$. Bajo estas definiciones, es fácil verificar que la solución clásica $Q_{C}(\bar{x})$ del problema del voceador maximiza $E[b(Y)]$, y la solución Bayesiana $Q_{B}(\bar{x})$ maximiza $E[b(Z)]$, donde la función $b$ está definida en (1). 
Tabla 1. Excesos de beneficio esperado y niveles de servicio del tamaño óptimo de pedido clásico para diferentes tamaños de muestra.

\begin{tabular}{|c|c|c|c|c|}
\hline \multirow{2}{*}{$n$} & \multicolumn{2}{|c|}{ Exceso de Beneficio } & \multicolumn{2}{c|}{ Nivel de Servicio } \\
\cline { 2 - 5 } & Media & Desv. Est. & Media & Desv. Est. \\
\hline 5 & 25.95 & 18.02 & 0.732 & 0.032 \\
\hline 10 & 13.61 & 6.13 & 0.770 & 0.025 \\
\hline 20 & 7.23 & 2.16 & 0.813 & 0.018 \\
\hline 50 & 3.10 & 0.59 & 0.861 & 0.011 \\
\hline 100 & 1.61 & 0.22 & 0.885 & 0.009 \\
\hline 150 & 1.08 & 0.12 & 0.894 & 0.008 \\
\hline 200 & 0.82 & 0.08 & 0.899 & 0.008 \\
\hline 250 & 0.66 & 0.06 & 0.901 & 0.008 \\
\hline 300 & 0.55 & 0.05 & 0.903 & 0.008 \\
\hline
\end{tabular}

Para $k=0,1, \ldots$ denotamos $p_{1}(k)=P[Y=k]$ y $p_{2}(k)=P[Z=k]$, y en la Fig. 4 presentamos la gráfica de la función $p_{2}(k)-p_{1}(k)$ para $k \geq 0$ y los mismos datos de la Fig. 1 , asumiendo $\bar{x}=0.5$, donde podemos apreciar que dicha función satisface la siguiente propiedad.

Propiedad 1. Para $n \geq 1, \quad \bar{x}>0$ y $T>0$ dados, y $g(y)$ como en (18), existen valores $0 \leq k_{1}<k_{2}<\infty$ tales que $g(y)>0$ para $0 \leq y \leq k_{1}, y \geq k_{2}$; mientras que $g(y) \leq 0$ para $k_{1}<y<k_{2}$.

A continuación mostramos que la Propiedad 1 siempre se cumple, para lo cual definimos

$$
g(y)=\ln \left[p_{2}(y) / p_{1}(y)\right]=\ln [\Gamma(n+y)]-\ln [\Gamma(n)]+n \ln (p)+y \ln (1-p)+\lambda-y \ln (\lambda)
$$

donde $\lambda=T / \bar{x}$ y $p=\bar{x} /(\bar{T}+\bar{x})$. Nótese que, a partir de $e^{\bar{T} / \bar{x}}>1+\bar{T} / \bar{x}$ se prueba fácilmente que $g(0)>0$, lo cual garantiza que existe $k_{1}$ tal que $g(y)>0$ para $0 \leq y \leq k_{1}$. Por otro lado, $\lim _{y \rightarrow \infty} g(y)=\infty$, lo que garantiza que que existe $k_{2}$ tal que $g(y)>0$ para $y \geq k_{2}$. Finalmente, $g^{\prime}(y)=\Psi(n+y)-\ln (a)$, donde $a=n / p$ y $\Psi(y)$ es la conocida función digamma, lo que muestra que $g(y)$ decrece para $y<y_{0}$ y crece para $y>y_{0}$, donde $y_{0}$ es la única solución de $\Psi(n+y)-\ln (a)=0$. La Propiedad 1 resulta entonces de la aplicación del Teorema del Valor Intermedio, haciendo uso de $\sum_{k=0}^{\infty} p_{1}(k)=\sum_{k=0}^{\infty} p_{2}(k)=1$.

La siguiente definición es bastante conocida, y la incluimos por completitud. Por otro lado, la siguiente Proposición es consecuencia directa de la Propiedad 1, el Teorema 2 de Rothschild y Stiglitz y de $E[Y]=E[Z]=T / \bar{x}$.

Definición 1. Se dice que una variable aleatoria $Y$ tiene dominancia estocástica de segundo orden sobre una variable aleatoria $Z$ si $E[U(Y)] \geq E[U(Z)]$ para cualquier función cóncava $U$.

Proposición 1. Sean $n \geq 1, \bar{x}>0$ y $T>0$ dados. Si $Y, Z$ son variables aleatorias distribuidas como Poisson con esperanza $\lambda=T / \bar{x}$ y como binomial negativa con parámetros $n$ y $p=\bar{x} /(\bar{T}+\bar{x})$, respectivamente, entonces $Y$ tiene dominancia estocástica de segundo orden sobre $Z$.

Corolario 1. Sean $n \geq 1, \quad \theta>0$ y $T>0$ dados, entonces $P\left[B_{C}\left(Q_{C} \mid x\right)-B_{B}\left(Q_{B} \mid x\right) \geq 0\right]=1$, donde $B_{C}\left(Q_{C} \mid x\right)$ y $B_{B}\left(Q_{B} \mid x\right)$ corresponden al ejemplo definido por la ecuación (17).

Este corolario es consecuencia directa de que la función $b$ definida en la ecuación (1) es cóncava y de que $P[\bar{X}>0]=1$, donde $\bar{X}$ es el promedio de una muestra aleatoria de tamaño $n$ de la distribución exponencial con esperanza $1 / \theta$. Este corolario muestra que, bajo condiciones muy generales para el ejemplo de la ecuación (17), el método clásico exagera el verdadero beneficio esperado (que corresponde al método Bayesiano).

\section{Estimación del Tamaño Óptimo de Pedido Usando Simulación}

Con la finalidad de ilustrar cómo se puede calcular el tamaño óptimo de pedido cuando la complejidad del modelo de pronóstico no permite la obtención de un resultado analítico, a continuación mostramos la aplicación del algoritmo MP para encontrar el tamaño de pedido óptimo propuesto utilizando simulación. 


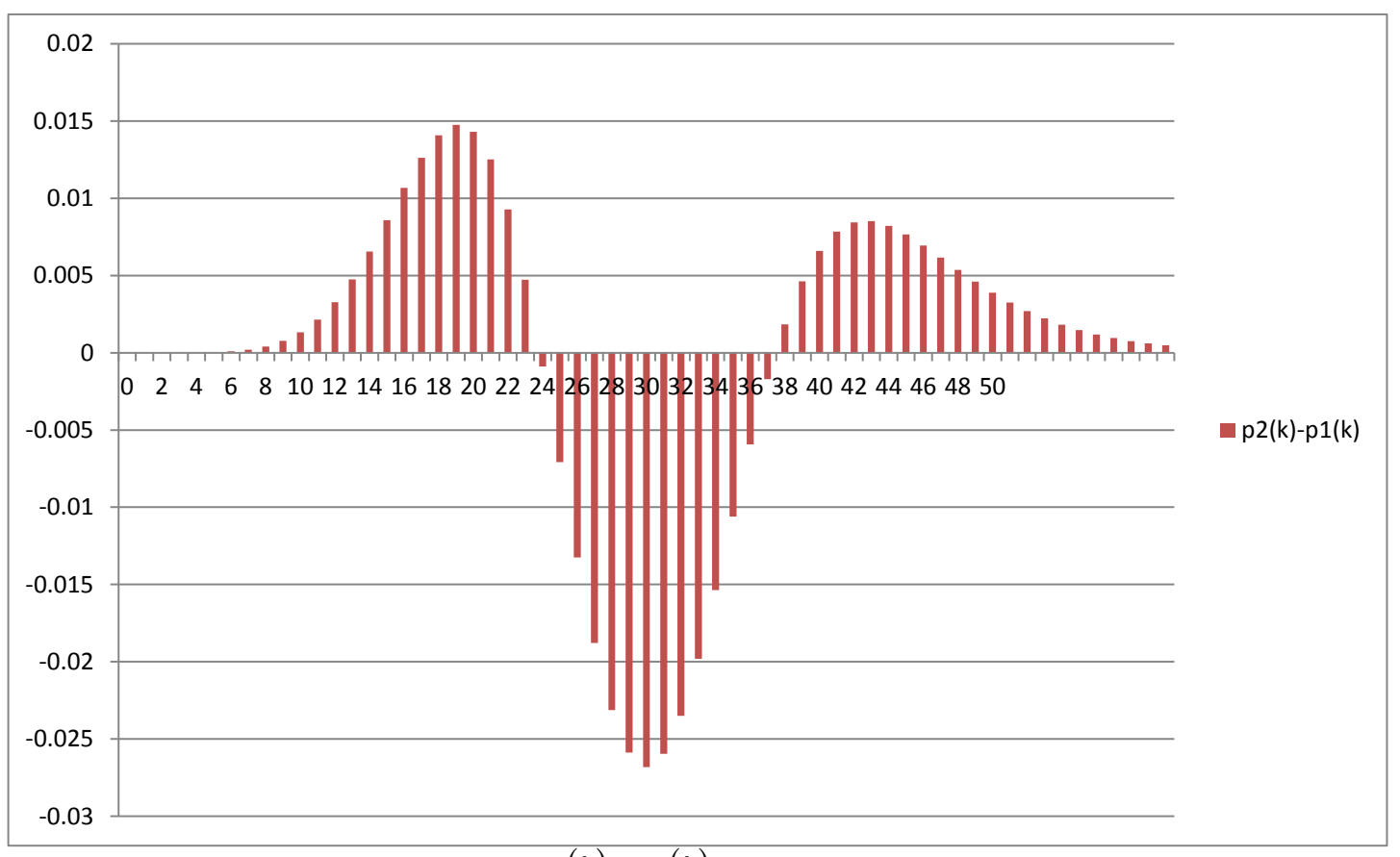

Fig. 4. Gráfica de la función $p_{2}(k)-p_{1}(k)$ para valores de $k$ enteros no-negativos.

Para aplicar el método MP a nuestro ejemplo, hemos considerado nuevamente los datos $T=15, n=20$, $\sum_{i=1}^{n} x_{i}=10, u=9, w=1$, para los cuales ya sabemos que el tamaño de pedido óptimo es de $Q_{\mathrm{B}=41}$, con un beneficio esperado de $B_{B}\left(Q_{B} \mid X\right)=253.38$. De acuerdo con el algoritmo descrito en la Fig. 2 de Muñoz y Muñoz (2009), el algoritmo MP consiste en simular $m$ observaciones $w_{1}, \ldots, w_{m}$ de la demanda. La observación wi se obtiene simulando primero el valor del parámetro, utilizando la distribución posterior $p(\theta \mid x)$, y luego simulando $w_{i}$ con el modelo de pronóstico (dado el valor del parámetro) que, en nuestro caso, corresponde al modelo (11). Para el caso en que la demanda admite función de densidad, el tamaño óptimo de pedido se obtiene aplicando el método de estimación de cuantiles, imponiendo el nivel de servicio $\alpha=u /(u+w)$. Sin embargo, para el caso discreto es conveniente aplicar el método descrito por las ecuaciones (8) y (9), reemplazando la fda $F_{B}(y \mid x)$ por la distribución empírica de las observaciones $\mathrm{W}_{1}, \ldots, \mathrm{W}_{\mathrm{m}}$. Como se puede apreciar de la Tabla 2, para $m=1000$ observaciones se obtiene el método MP proporciona el tamaño óptimo de pedido de 41 , estimando el beneficio óptimo entre 247.9 y 257.08 , que cubre al verdadero valor (253.38). Para $m=100$, el número de observaciones es insuficiente para obtener el tamaño óptimo de pedido (curiosamente no se obtuvo ninguna observación de valor 43). Para valores $m>1000$ el método MP debería seguir proporcionando el tamaño óptimo de pedido de 41 , con una estimación más precisa del beneficio esperado.

Con base en los resultados obtenidos, se recomienda utilizar el método propuesto cuando el tamaño de las observaciones no es muy alto, debido a que, en este caso, la incertidumbre paramétrica es significativa. Por otro lado, si se utiliza la simulación estocástica para estimar el tamaño óptimo de pedido, debe considerarse un número de observaciones simuladas suficientemente grande para asegurar una precisión adecuada.

Tabla 2. Resultados de la aplicación del método MP para $m=100$ y $m=1000$.

\begin{tabular}{|c|c|c|c|c|c|}
\hline \multirow{2}{*}{$\mathrm{m}$} & \multicolumn{3}{|c|}{ Tamaño Óptimo } & \multicolumn{3}{c|}{ Estimación del Beneficio Esperado } \\
\cline { 4 - 6 } & \multicolumn{2}{|c|}{} & Puntual & Lim. Inf. & Lim. Sup. \\
\hline \multirow{2}{*}{100} & $d_{k}$ & 42 & 256.00 & 240.65 & 271.35 \\
\cline { 2 - 6 } & $d_{k+1}$ & 44 & 256.20 & 240.14 & 272.26 \\
\hline \multirow{2}{*}{1000} & $d_{k}$ & 40 & 252.37 & 247.89 & 256.85 \\
\cline { 2 - 6 } & $d_{k+1}$ & 41 & 252.49 & 247.90 & 257.08 \\
\hline
\end{tabular}

\section{CONCLUSIONES}

Los resultados que se obtuvieron al experimentar con el método propuesto, así como los correspondientes resultados analíticos, muestran que el método clásico proporciona pronósticos exagerados del beneficio esperado. Por otro lado, los resultados con ambos métodos tienden a coincidir, a medida que el número de observaciones reales crece. 


\section{AGRADECIMIENTOS}

Este trabajo se ha desarrollado con el apoyo de la Asociación Mexicana de Cultura A.C. y del Consejo Nacional de Ciencia y Tecnología (CONACYT) de México. Los autores manifiestan su agradecimiento al Editor y a un Árbitro anónimo por sus valiosos comentarios y, en particular, la bibliografía sugerida, que han permitido mejorar, en forma y en fondo, el contenido de este artículo.

\section{REFERENCIAS}

Agrawal, V., y S. Seshadri, "Impact of Uncertainty and Risk Aversion on Price and Order Quantity in the Newsvendor Problem", Manufacturing \& Service Operations Management 2, 410-423 (2000)

Azouri K. S., "Bayes Solution to Dynamic Inventory Models under Unknown Demand Distribution", Management Science 31, 1150-1160 (1985)

Bernardo, J. M. y A. F. M. Smith, "Bayesian Theory”, Wiley, Chichester (2009)

Berger, J. O y J. M. Bernardo, "Ordered group reference priors with application to the multinomial problem", Biometrika 79, 25-37 (1992)

Chen, L. y E. L. Plambeck, "Dynamic Inventory Management with Learning About the Demand Distribution and Substitution Probability", Manufacturing Service and Operations Management 10, 236-256 (2008)

Chen, L., "Bounds and Heuristics for Optimal Bayesian Inventory Control with Unobserved Lost Sales", Operations Research 58, 396-413 (2010)

Cheng, R. C. H. y W. Holland, "Calculation of Confidence Intervals for Simulation Output", ACM Transactions on Modeling and Computer Simulation 14, 344-362 (2004).

Chung, K. L., "A Course in Probability Theory" (3a. ed.), Academic Press, San Diego (2001)

Eppen, G. D. y A. V. Iyer, "Improved Fashion Buying with Bayesian Updates", Operations Research 45, 805819 (1997)

Jain, A., N. Rudi, and T. Wang, "Demand Estimation and Ordering Under Censoring: Stocking-Out Timing is (Almost) All You Need", Operations Research 63, 134-150 (2015)

Keren, B. y J. S. Pliskin, "A Benchmark Solution for the Risk-Averse Newsvendor Problem", European Journal of Operational Research 174, 1643-1650 (2006)

Lariviere, M. A. y E. L. Porteus, "Stalking Information: Bayesian Inventory Management with Unobserved Lost Sales", Management Science 45, 343-363 (1999)

Levi R., R. Roundy y D. B. Shmoys, "Provably Near-Optimal Sampling Based Policies for Stochastic Inventory Control Models", Mathematics of Operations Research 32, 821-839 (2007)

Levi, R, G. Perakis y J. Uichanco, "The Data-Driven Newsvendor Problem: New Bounds and Insights", Operations Research 63, 1294-1306 (2015)

Limón, J., M. A. Rodríguez, J. Sánchez y D. A. Tlapa, "Metodología Bayesiana para la Optimización Simultánea de Múltiples Respuestas", Información Tecnológica 23, 151-166 (2012)

Muñoz, D.F., "Administración de Operaciones Enfoque de Administración de Procesos de Negocios", Cengage, México (2009).

Muñoz Negrón, D. F. y D. F. Muñoz Medina, "Pronósticos Bayesianos para Repuestos de Automóviles usando Simulación Estocástica”, Journal of Economics, Finance and Administrative Science 14, 7-20 (2009)

Muñoz, D.F., D.G. Muñoz y A. Ramírez-López, "On the Incorporation of Parameter Uncertainty for Inventory Management using Simulation", International Transactions in Operational Research 20, 493-513 (2013)

Rothschild, M. y J. E. Stiglitz, "Increasing Risk: I. A Definition”, Journal of Economic Theory 2, 225-243 (1970)

Scarf, H. (1959), "Bayes Solutions of the Statistical Inventory Problem", The Annals of Mathematical Statistics 30, 490-508 (1959)

Silver, E. A., "Bayesian Determination of the Reorder Point of a Slow Moving Item", Operations Research 13, 989-997 (1965) 